\title{
Effects of maternal exposure to a bacterial antigen and altered post-hatching rearing conditions on avian offspring behaviour
}

\author{
Rafał Martyka $^{1}$ (D) Ewa B. Śliwińska ${ }^{2}$ (D) Piotr Tryjanowski ${ }^{2}$ (I) \\ Received: 20 March 2020 / Revised: 9 February 2021 / Accepted: 12 February 2021 / Published online: 26 February 2021 \\ (C) The Author(s) 2021
}

\begin{abstract}
The early-life environment plays a crucial role in shaping morphological, physiological, and behavioural traits, with potential long-term consequences for fitness. Indeed, a set of factors experienced by offspring during prenatal and early post-natal development has been recognised to affect behavioural trait expression in later life. Several studies have shown that in birds, nutritional and social rearing conditions and maternal and/or neonatal immunisations may profoundly determine the development and establishment of behaviour in offspring. To our knowledge, no research has examined whether and how the interaction between immune-mediated maternal effects and post-hatching rearing conditions affects offspring behaviour. Here, we studied the effects of maternal exposure to a bacterial antigen and altered brood size on docility, breathing rate, and aggression in the offspring of great tit, Parus major. We used a $2 \times 2$ design to investigate the interactive effects of maternal immunisation and brood size manipulation on offspring behavioural development. We found no such interactive effect on offspring behaviour, although we observed it regarding to offspring body mass and tarsus length. Maternal immunisation itself did not affect offspring behaviour. However, we demonstrated that the offspring breathing rate and level of aggression were affected by brood size manipulation. Both breathing rate and aggression in offspring reared in enlarged broods were lower than those in offspring reared in non-manipulated broods. Our study did not confirm earlier reports that immune-mediated maternal effects modulate offspring behavioural development, but we showed that brood size during rearing might indeed be a factor that affects offspring behaviour.
\end{abstract}

\section{Significance statement}

The early environment experienced by offspring constitutes a significant source of developmental plasticity, which may profoundly affect the establishment of their behavioural traits. Food availability, social conditions, and maternal or offspring infection are crucial factors shaping various behavioural traits in birds. However, there remains a lack of studies emphasising the potential interactive effects of early-life conditions on behavioural trait development in natural bird populations. Here, to our knowledge for the first time, we experimentally examined how maternal immunisation and altered post-hatching rearing conditions interact to determine the behaviour of fledged offspring. We found that maternal treatment and brood size manipulation interactively affected offspring body mass and tarsus length, but this interaction had no effect on offspring behaviour. Our findings suggest that different mechanisms may underlie the development of morphological and behavioural traits.

Keywords Adaptive predictive phenotype shaping · Behavioural development · Brood size manipulation · Immune-mediated maternal effects · Lipopolysaccharide · Parus major

Communicated by K. van Oers

Rafał Martyka

martyka@iop.krakow.pl

1 Institute of Nature Conservation, Polish Academy of Sciences, Mickiewicza 33, 31-120 Kraków, Poland

2 Institute of Zoology, Poznań University of Life Sciences, Wojska Polskiego 71C, 60-625 Poznań, Poland

\section{Introduction}

Animals express many different behaviours that allow them to cope with environmental challenges, thereby enabling them to survive and reproduce (Langenhof and Komdeur 2018). The development of behavioural traits is a product of the interaction between genetic and environmental factors (e.g. Drent et al. 2003; Stamps and Groothuis 2010; Brommer and Kluen 2012; Carere and Maestripieri 2013). In particular, the 
early-life environment is believed to be crucial for the establishment of behavioural phenotypes because it provides primary cues for the induction of developmental plasticity (Bateson et al. 2014; Groothuis and Taborsky 2015), which in turn generates variation in behaviour among individuals and ultimately affects their fitness (Lindström 1999; Riebel et al. 2012; Kasumovic 2013; Careau et al. 2014; Langenhof and Komdeur 2018). The earliest extrinsic environmental cues experienced by offspring are commonly provided by parents, especially mothers, via environmentally induced pre- and post-natal maternal (parental) effects (Mousseau and Fox 1998). Nevertheless, direct environmental cues encountered early in life by individuals, such as social conditions, may also be crucial to evoke developmental plasticity (Lindström 1999; Fischer et al. 2015). Such early-life programming of individuals, both caused by maternal or direct environmental effects, may be adaptive if phenotypic changes initiated during development increase fitness benefits in later life (Mousseau and Fox 1998; Lindström 1999; Groothuis and Taborsky 2015). However, adaptive phenotypic shaping of behavioural traits is expected to occur only when the developmental environment provides a forecast of future conditions; otherwise, a mismatch between the developmental conditions and the environment experienced later in life can result in maladaptive effects on behavioural phenotypes (Bateson et al. 2014; Groothuis and Taborsky 2015).

In birds, there are three main groups of developmental cues that have a potential for programming offspring behaviour. The first group includes post-natal nutritional conditions, from which the quality and quantity of food available to growing nestlings seem to be crucial for neurodevelopment, and, consequently, behavioural trait expression (Carere et al. 2005; Krause and Naguib 2011; Noguera et al. 2015; van Oers et al. 2015). For example, nestlings of the great tit, Parus major, reared under conditions of food scarcity, became more aggressive and explorative in later life (Carere et al. 2005). In turn, a study on the zebra finch, Taeniopygia guttata, showed that a low-quality diet in terms of micronutrients provided during development results in reduced boldness among male offspring and increased aggressiveness among female offspring (Noguera et al. 2015). The second group of developmental cues includes social conditions occurring in a nest, such as the number of competing nestlings and brood sex ratio, which have found to be significant to modify offspring aggression, stress responses, and exploratory behaviour (Carere et al. 2005; Naguib et al. 2011). The third group includes the activation of the immune system in developing offspring, resulting from an antigenic challenge or parasite exposure (e.g. Bischoff et al. 2009; Butler et al. 2012; Grindstaff et al. 2012). In mallards, Anas platyrhynchos, Butler et al. (2012) found that immunisation of chicks with a non-pathogenic antigen (sheep red blood cells) enhanced the activity of mature offspring in a novel environment. In another study on zebra finches, male but not female nestlings immunised with lipopolysaccharide (LPS) during the growth period became slower learners when they were adult birds (Grindstaff et al. 2012). Interestingly, the same authors have also shown that maternal immune challenge and offspring developmental immunisation interact, affecting neophobia of adult birds in a sex-specific manner. Thus, sons that were immunised with a different antigen than their mothers (mismatching effect) exhibited the least neophobia among the overall offspring (Grindstaff et al. 2012). There is increasing evidence that both maternal and offspring immunisations can affect the neurodevelopmental processes, resulting in subsequent behavioural alterations in adult life (Grindstaff 2016; Boulanger-Bertolus et al. 2018).

Although the number of studies dealing with the consequences of early-life conditions in shaping behavioural traits is increasing, a full understanding of processes linked to the development and establishment of the behaviour of individuals is still lacking (Stamps and Groothuis 2010; Groothuis and Taborsky 2015; Trillmich et al. 2018). Despite growing evidence that post-natal rearing conditions and maternal and/ or offspring immune challenges constitute significant developmental cues for eliciting ontogenic changes in bird behaviour (Groothuis and Taborsky 2015; Grindstaff 2016), to our knowledge, no study has assessed the interactive effects of maternal immunisation and altered post-natal rearing conditions on the expression of behavioural traits in avian offspring.

To examine whether maternal immune challenge and postnatal rearing conditions affect offspring behavioural traits (i.e. docility, breathing rate, and aggression) and whether the effects are independent or interactive, we performed an experiment in a natural population of great tits using a $2 \times 2$ factorial design. First, half of the females were immunised with LPS to simulate bacterial infection, and the other half received a saline injection (control group). LPS is an element of the outer membranes of gram-negative bacteria that induces an acutephase response with fever and behavioural changes, such as lethargy and anorexia (Hart 1988). It also stimulates the production of proinflammatory cytokines (Klasing and Leshchinsky 1999) and the activation of the hypothalamicpituitary-adrenal (HPA) axis (Karrow 2006). Moreover, maternal immunisation with LPS results in the production of LPS-specific antibodies, which are then deposited into the eggs and utilised by nestlings (Sunwoo et al. 1996; Merrill and Grindstaff 2014). Two days after hatching, we partially cross-fostered nestlings among the broods of immunised and control females. Simultaneously, to alter post-natal rearing conditions, we enlarged half of the broods of immunised and control females, and the second half remained unmanipulated (control). We also immunised all 5-day-old nestlings with LPS to activate their immune system during development.

It has been documented that nestling immune challenge with LPS during development suppresses offspring growth 
(Klasing and Leshchinsky 1999; Grindstaff 2008) and impairs learning ability in adult birds (Grindstaff et al. 2012). However, LPS-specific antibodies passed onto nestlings due to maternal immunisation may partially ameliorate the growth-suppressive effects of offspring exposure to LPS (Grindstaff 2008). This may allow LPS-challenged offspring to eliminate the bacterial antigen without an intense stimulation of a costly innate immune response linked to the production of proinflammatory cytokines (Klasing and Leshchinsky 1999). Thus, offspring of LPS-immunised females are expected to better cope with exposure to LPS, avoiding excessive activation of the HPA axis, which should modify the potential effects of such developmental immunisation on behavioural trait expression. Therefore, we predicted that offspring of control and immunised mothers would differ in behaviour; however, we had no specific predictions regarding the direction of such behavioural changes. Furthermore, we expected that offspring reared in enlarged broods will exhibit higher breathing rates and aggression and lower docility than offspring reared in control broods. Indeed, earlier studies have shown that an increase in among-sibling competition and poorer nutritional conditions during development enhanced offspring exploratory behaviour (a trait linked to breathing rate, see Fucikova et al. 2009) and aggression in adult life, which may result from adaptive programming, which prepares them for similar conditions later in life (Carere et al. 2005; Naguib et al. 2011). We also predicted that an immune-mediated maternal effect and altered post-hatching rearing conditions should interactively shape offspring behavioural expression. Previous studies have documented such interactive effects in relation to the development of morphological and physiological traits, indicating that fitness consequences of pre-natal maternal effects may depend on post-natal environmental context (Ismail et al. 2015; Martyka et al. 2018). Therefore, we searched for a significant interaction between maternal immunisation and brood size manipulation to explain variation in offspring behaviour.

\section{Material and methods}

\section{Study area and model species}

A population of great tit breeding in nest boxes in the northern part of the Niepołomice Forest, southern Poland (50 $06 \mathrm{~N} 20^{\circ}$ $24 \mathrm{E}$ ), was the subject of this research. The study area was located in a deciduous forest dominated by oak, hornbeam, and lime trees. In total, there were 233 wooden nest boxes (with interior dimensions: $9.0 \times 9.0 \times 27.5 \mathrm{~cm}$ ) on the study plot, approximately distributed in a $50 \times 40 \mathrm{~m}$ grid. The great tit is a small, sexually dimorphic, cavity-nesting bird commonly used as a model in behavioural research (Fucikova et al. 2009; Naguib et al. 2011; Laine et al. 2016). Great tit females from the studied population produced 1-2 clutches during a single breeding season. Egg incubation in this species lasts approximately 13 days, and after hatching, nestlings are fed by both parents and fledge within the next 15-18 days (Gosler 1993).

\section{General field procedures}

At the beginning of April 2014, we systematically monitored nest boxes to determine the laying date and clutch size of all breeding great tit females. When females completed egg laying, we captured them within $3.5 \pm 0.1$ (mean \pm SE) days after the onset of incubation. We then assigned captured females to one of two groups: experimental or control. On the day of capture, females from the experimental group $(N=24)$ were intra-abdominally injected with $50 \mu \mathrm{L}$ of LPS (from the Salmonella enterica serotype Typhimurium; Sigma, Cat. No. L-7261) suspended in phosphate-buffered saline (PBS) at a concentration of $0.1 \mathrm{mg} \mathrm{kg}$ body mass $^{-1}$ (e.g. Grindstaff et al. 2006), whereas females from the control group $(N=$ 29) received $50 \mu \mathrm{L}$ of PBS. On the same day, we measured female body mass (to the nearest $0.01 \mathrm{~g}$ using an electronic balance) and individually marked her using numbered aluminium and alphanumeric rings. Females assigned to the experimental and control groups did not differ in body mass (twosample $t$-test: $t_{51}=0.158, P=0.88$ ) or clutch size (two-sample $t$-test: $\left.t_{51}=0.038, P=0.97\right)$ on the day of capture. We then removed the nest together with eggs from the nest box to force females to repeat breeding.

We located and regularly visited replacement nests to indicate the beginning of egg laying and clutch size for the next step. To identify re-nested females, we used alphanumeric rings, which allowed us to easily recognise each female during her stay in a nest box. In total, 40 females started laying replacement clutches, including 24 control and 16 immunised females. Finally, we used 31 broods (16 of control and 15 of immunised females) in our analyses, whereas nine broods served as donor nests (for details, see below). We then established hatching dates by inspecting nest boxes with repeated clutches around the expected hatching date. We performed cross-fostering to control for potentially confounding effects of maternal immunisation on parental provisioning and separate prenatal maternal effects from post-hatching rearing conditions. On the second day after hatching (hatching day = day 0), we swapped half of the nestlings among pairs of broods belonging to immunised and control females. We matched those dyads only when they had the same hatching date and clutch size ( \pm 1 egg). Before cross-fostering, we measured the body mass of all nestlings and ranked them in relation to their body mass. Next, we swapped every second nestling based on their body mass rank such that crossfostered nestlings represented all mass hierarchies observed within the original broods (Brommer and Kluen 2012; van Oers et al. 2015). We could not perform cross-fostering for 
all replacement nests because there were not enough broods of immunised and control females with the same hatching date or a similar clutch size, and consequently, others could not be matched. Consequently, our dataset included 18 crossfostered and 13 non-cross-fostered broods (six from immunised females and seven from control). On day 2 after hatching, we found no differences in log-transformed nestling body mass (paired $t$-test: $t_{17}=-0.540, P=0.60$ ) or brood sex ratio (paired $t$-test: $t_{15}=0.098, P=0.92$ ) within the same nest, before and after cross-fostering. The log-transformed body mass of 2-day-old nestlings did not differ among siblings that stayed in their original broods and those swapped to foster broods (linear mixed model [LMM] with female identity as a random factor and nestling status, moved vs. not moved, as a fixed factor: $\left.F_{1,129.8}=0.10, P=0.76\right)$. Additionally, there were no differences in log-transformed body mass on day 2 (LMM with female identity and foster female identity as random factors and brood status, cross-fostered vs. non-crossfostered, as a fixed factor: $F_{1,26.7}=0.03, P=0.87$ ) or brood sex ratio (two-sample $t$-test: $t_{25}=-0.944, P=0.35$ ) between cross-fostered and non-cross-fostered nests (after crossfostering was performed).

On day 2 after hatching, shortly after performing crossfostering, we also manipulated brood size in nests of immunised and control females. To obtain a standardised difference in rearing conditions between them, we added three extra nestlings originating from donor nests to randomly chosen broods within a dyad, with the second remaining unmanipulated (Dubiec et al. 2006; Arct et al. 2013). We performed the same treatment among non-cross-fostered nests; half of the broods of immunised and control females were enlarged, whereas the second half were unmanipulated. The extra nestlings originated from nine donor nests that were non-crossfostered broods of eight control females and one immunised female. Extra nestlings came from broods with a similar hatching date as enlarged broods, and their body mass was within the range of body mass recorded among nestlings in the brood being enlarged. Neither additional nestlings nor donor broods were included in the analyses. After cross-fostering and brood size manipulation, we had four groups of offspring: nestlings of control females reared in non-manipulated (control) broods, nestlings of control females reared in enlarged broods, nestlings of immunised females reared in non-manipulated (control) broods, and nestlings of immunised females reared in enlarged broods. There were no differences among the four groups of offspring, either in the case of log-transformed body mass on day 2 (LMM with female identity and foster female identity as random factors, and maternal immunisation and brood size manipulation, as well as their interaction, as fixed factors: $F_{1,184.8}=0.01, P=$ 0.91 ) or brood sex ratio (GLM: $F_{1,23}=0.78, P=0.39$ ), as revealed by the non-significant interaction between maternal immunisation and brood size manipulation.

\section{Procedures for nestlings}

We measured nestling body mass and individually marked them by clipping their nails 2 days after hatching. We repeated nail clipping in 5-day-old nestlings (the same code used), which allowed us to identify and ring the nestlings on day 14. On day 5 after hatching, we also intra-abdominally injected all nestlings with $25 \mu \mathrm{L}$ of LPS suspended in PBS with a concentration of $0.1 \mathrm{mg} \mathrm{kg}$ body mass $^{-1}$ (see Grindstaff et al. 2006). When nestlings reached 14 days after hatching, we again determined the nestling body mass and measured their tarsus and wing length. Body mass was measured to the nearest $0.01 \mathrm{~g}$ using an electronic balance, the tarsus was measured to the nearest $0.1 \mathrm{~mm}$ using an electronic calliper, and the wing was measured to the nearest $1 \mathrm{~mm}$ using a ruler. All nestlings were treated as similarly as possible during handling.

\section{Offspring behaviour assays}

To quantify behavioural traits in fledged offspring, i.e. docility, breathing rate, and aggression, we used a previously applied protocol by Brommer and Kluen (2012). On day 14 after hatching, we took all nestlings from the nest box individually and placed each in an individually numbered paper bag (with dimensions: $16.5 \times 28 \times 6 \mathrm{~cm}$ ). When all nestlings were placed in their paper bags, we mixed the order of all bags to randomise further handling during behavioural tests. Next, we opened each paper bag and measured offspring behaviour. Briefly, a nestling was taken from the bag and immediately placed on the palm of the observer's hand in a specific position wherein the nestling was laid on its back, with its neck held between the observer's index and middle finger, and one leg held by the foot between the thumb and index finger, while the second leg was held by the middle and ring fingers. The secured nestling was placed at an approximate distance of $40 \mathrm{~cm}$ from the observer's face. The stopwatch was on, and the number of nestling struggles was counted for a period of $10 \mathrm{~s}$. Finally, the number of struggles per second was multiplied by -1 to ensure that lower scores reflected less docile offspring (wigglier). Docility is a measure that may indicate the obstinacy of an individual (David et al. 2011) and also reflects susceptibility to predation (Møller et al. 2011). After ending the docility measurement, we immediately evaluated the breathing rate while still keeping the nestling in the same position. The observer measured the time required to take 30 breaths using the lap time function. This assay was performed twice (without delay) to obtain two measurements. The breathing rate was estimated using the average of those measurements and expressed as the number of breaths per second. Most studies assume a positive association between breathing rate and stress experienced by individuals during handling, suggesting that breathing rate is a good measure of stress in 
birds (Carere and van Oers 2004; Naguib et al. 2011; van Oers et al. 2015). Moreover, nestling handling stress, measured by counting breaths, is a positive predictor of exploratory behaviour in adult great tits (Fucikova et al. 2009). The last test of handling aggression was performed during ringing and morphological measurements of nestlings (see 'Procedures for nestlings'). During that time, lasting approximately $3 \mathrm{~min}$, we quantified nestling aggression using a Likert-scale score that determines the extent of aggressive responses of birds, such as picking and/or struggling when an individual is handled. This index score ranges from 1 , indicating an individual that is passive, to 5 for an individual that picks and struggles during the entire handling time (for more details, see Brommer and Kluen 2012). All behavioural measurements were performed by RM. It was not possible to record data blind because our study involved focal animals in the field.

\section{Nestling sex determination}

To obtain DNA samples for offspring sex determination, we used blood samples collected from 5-day-old nestlings, tissue samples taken from dead nestlings (until day 5), and embryos (unhatched eggs). Offspring sex was successfully determined in 234 of 239 collected samples from live and dead nestlings and all embryos (13). Blood and tissue samples were kept in $96 \%$ ethanol $(0.5 \mathrm{~mL})$ and stored at room temperature until analysis. DNA was extracted with Chelex 5\% (SigmaAldrich, Saint Louis, MO, USA), and sexing was performed by the amplification of two homologous genes ( $C H D 1-W$ and CHD1-Z) following the protocol described by Griffiths et al. (1998).

\section{Statistical analyses}

We fitted a generalised linear model with binomial error variance and a logit link function to assess the probability of renesting among control and immunised females. To compare differences between control and immunised females in relation to the time required for a female to re-nest, clutch size of replacement nests, hatching success (the proportion of hatched eggs within a clutch), and primary brood sex ratio (the proportion of males within a clutch, including unhatched eggs and dead nestlings), we performed two-sample $t$-tests, with correction for unequal variances when necessary. Pearson's correlation tests were used to determine the relationships between the examined behavioural traits. To analyse body mass on days 2 and 14 after hatching, the length of the tarsus and wing and behavioural traits in fledged offspring, we fitted LMMs using the lme4 package (Bates et al. 2015). A model that analysed nestling body mass on day 2 included maternal immunisation (control vs. immunised females) and offspring sex (females vs. males, to control for potential sex-specific effects) as categorical fixed factors. Models analysing the remaining dependent variables included the following categorical fixed factors: maternal immunisation, brood size manipulation (control vs. enlarged broods), offspring sex, and measure order (factor with 11 levels, only in analyses of offspring behaviour). Measure order reflected the sequence in which offspring were measured during behavioural tests and served to control for the period when offspring were isolated from siblings. Such isolation might be stressful for nestlings; thus, it may potentially affect the results of behavioural measurements (Brommer and Kluen 2012). Nestling body mass on day 2, divided into two standardised covariates representing between- and within-brood effect (see van de Pol and Wright 2009), were introduced in all models to control for the effect of early-life conditions on traits measured at 14 days of age. In models analysing behavioural traits, we added offspring body mass on day 14 (also as two standardised covariates attributed to between- and withinbrood effect, see van de Pol and Wright 2009) to account for an individual's condition on the day of behavioural measurements. There was no problem with collinearity when body mass on days 2 and 14 was analysed in the same models (variance inflation factor $<2$ ). In all LMMs, we also tested the following two-way interactions: maternal immunisation $x$ brood size manipulation (a primary interest), maternal immunisation $\times$ offspring sex, and brood size manipulation $\times$ offspring sex (to test for potential sex-specific effects of the treatments). To reduce the initial models, we dropped covariates and interactions with $P>0.05$, starting with the least significant terms. Only marginally non-significant effects (close to 0.05 ) were retained because of a better model fit. The nest of origin (female identity) and the nest of rearing (female foster identity) were included in all LMMs as random factors, except for the model examining nestling body mass on day 2 (only female identity included). To obtain the significance of random factors, we performed a likelihood ratio test implemented in the lmerTest package (Kuznetsova et al. 2017). We also partitioned variance components for each final model to determine how much variance was explained by the nest of origin and rearing among the examined morphological and behavioural traits. For this purpose, we used the insight package (Lüdecke et al. 2019), which allowed to estimate variance for all model terms, i.e. fixed factors (pulled), each random factor, and residuals. All models were checked for normality and heteroscedasticity of residuals, and if necessary, a dependent variable was transformed. To examine fixed effects in LMMs, we performed $F$-tests, in which the degrees of freedom were approximated by the Kenward-Roger method, using the pbkrtest and lmerTest packages (Halekoh and Hojsgaard 2014; Kuznetsova et al. 2017). To perform post hoc pairwise contrasts of the least square means for factors involved in interactions, we used the emmeans package (Lenth 2020). Moreover, the marginal and conditional $R^{2}$ for LMMs were calculated using the MuMIn package (Bartoń 
2020). The significance level of all tests was set at $P \leq 0.05$. Sample sizes differed among analyses because of female nest abandonment, nest predation, or missing measurements. The results are presented as the raw mean $\pm \mathrm{SE}$. All analyses were performed in the R environment ( $R$ Core Team 2020).

\section{Results}

\section{Effects of immunisation on maternal reproductive investment and early body mass of nestlings}

The probability of re-nesting did not differ between immunised and control females (Wald $\chi_{1}^{2}=1.79, P=0.18$; $N=53$ ). Control and immunised females began repeat breeding at a similar time $\left(t_{16.1}=-1.143, P=0.27 ; N=31\right)$, and they also did not differ in the size of replacement clutches $\left(t_{29}\right.$ $=0.048, P=0.96 ; N=31$ ). Maternal immunisation did not influence hatching success $\left(t_{29}=-0.734, P=0.27 ; N=31\right)$ or primary brood sex ratio $\left(t_{27}=0.359, P=0.72 ; N=29\right)$. The body mass of 2-day-old nestlings did not differ between control and immunised females, and it also did not depend on offspring sex (Table 1). The nest of origin explained 13\% of the variation in body mass on day 2 (Table 1).

\section{Effects of maternal and brood treatments on offspring conditions}

The body mass of 14-day-old nestlings was affected by an interaction between maternal immunisation and brood size manipulation and marginally by an interaction of maternal immunisation and nestling sex, and by the within-nest effect of nestling body mass on day 2 (Table 1). The first interaction showed that nestlings reared in unmanipulated (control) broods were significantly heavier than those reared in enlarged broods if they originated from control females (post hoc contrast: $t$-ratio $21.1=3.453, P=0.002$; Fig. 1 ). This effect was absent in the case of offspring originating from immunised females (post hoc contrast: $t$-ratio $24.3=1.513, P$ $=0.14$; Fig. 1). Moreover, offspring of immunised females tended to be heavier than offspring of control females when reared in enlarged broods (post hoc contrast: $t$-ratio ${ }_{14.0}=-$ $2.137, P=0.051$; Fig. 1 ), but there was no significant difference between the offspring of immunised and control females reared in control broods (post hoc contrast: $t$-ratio ${ }_{19.7}$ $=1.092, P=0.29 ;$ Fig. 1 ). The latter interaction was caused by a difference in body mass between male and female nestlings that was larger among offspring originated from immunised mothers (males vs. females: $17.4 \pm 0.3$ and $16.0 \pm 0.3 \mathrm{~g}$; post hoc contrast: $t$-ratio $\left.{ }_{139.0}=-4.294, P<0.001\right)$ than among those originated from control mothers (males vs. females: 16.7 \pm 0.2 and $16.0 \pm 0.3 \mathrm{~g}$; post hoc contrast: $t$ - ratio $_{136.0}=-2.388$, $P=0.018$ ). Offspring of the same sex originating from immunised and control females did not differ in body mass (post hoc contrast for males: $t$-ratio ${ }_{11.2}=-1.456, P=0.17$; post hoc contrast for females: $t$-ratio $18.1=0.513, P=0.61$ ). At 14 days after hatching, the offspring body mass variation was mainly attributed to the rearing nest $(40 \%$, Table 1$)$. In contrast, a small part of the variation was determined by the nonsignificant effect of the nest of origin (only 4\%, Table 1).

Nestling tarsus length on day 14 was influenced by an interaction between maternal immunisation and brood size manipulation, offspring sex, and the within-nest effect of body mass on day 2 (Table 1). The interaction was caused by a difference in tarsus length between offspring originating from control and immunised females when reared in control broods (post hoc contrast: $t$-ratio $27.4=3.245, P=0.003$; Fig. 2 ), but not when they were reared in enlarged broods (post hoc contrast: $t$-ratio $22.9=0.090, P=0.93$; Fig. 2). Additionally, nestlings of control females reared in control broods had longer tarsi compared to those reared in enlarged broods (post hoc contrast: $t$-ratio $26.5=2.063, P=0.049$; Fig. 2 ), with no such effect among nestlings of immunised females (post hoc contrast: $t$-ratio $35.2=-1.024, P=0.31$; Fig. 2 ). Male offspring had longer tarsi than female offspring on day 14 after hatching ( $20.0 \pm 0.1$ vs. $19.5 \pm 0.1 \mathrm{~mm}$; Table 1$)$. The variation in tarsus length on day 14 was determined by the nest of rearing (14\%), with less variation attributed to the non-significant effect of the nest of origin $(6 \%$, Table 1$)$.

Offspring reared in enlarged broods had a shorter wing length than offspring reared in control broods $(45.9 \pm 0.4 \mathrm{vs}$. $47.9 \pm 0.4 \mathrm{~mm}$, respectively; Table 1$)$. Male offspring had longer wings than female offspring $(47.6 \pm 0.4$ vs. $45.8 \pm$ $0.5 \mathrm{~mm}$, respectively; Table 1). The within-nest effect of body mass on day 2 positively predicted offspring wing length on day 14 after hatching (Table 1). The nest of origin and rearing nest explained a similar proportion of the total variance in wing length at 14 days of age, i.e. $10 \%$ and $16 \%$, respectively (Table 1).

\section{Effects of maternal and brood treatments on offspring behaviour}

Post-hatching brood size manipulation affected the breathing rate and aggression of offspring, but a similar effect was not found with respect to docility (Table 2). Contrary to brood treatment, we failed to detect any effect of maternal immunisation on the behavioural traits of the offspring (Table 2). Offspring reared in enlarged broods exhibited a lower breathing rate than offspring reared in control broods (Table 2, Fig. 3). Moreover, female offspring tended to breathe at a higher rate than male offspring, regardless of treatment $(1.682 \pm 0.039$ vs. $1.675 \pm 0.033$ breaths per sec., respectively; Table 2). The breathing rate was also positively correlated with the body mass of 14-day-old nestlings (as the within-nest effect only, Table 2). The breathing rate variance 
Table 1 Results of linear mixed models analysing body mass on days 2 and 14 after hatching, and tarsus and wing length on day 14 in offspring. Full models included maternal immunisation (control vs. immunised females), brood size manipulation (control vs. enlarged broods), and offspring sex (females vs.males) as categorical fixed factors. Moreover, standardised between- and within-nest effects of nestling body mass on day 2 were entered into models as covariates (except for the analysis of body mass on day 2). Nest of origin and rearing nest were introduced in all models as random factors (estimates of each variance component are shown accompanied with CIs and the proportion of variance explained). Presented are reduced (final) models after removing non-significant covariates and two-way interactions among maternal immunisation, brood size manipulation, and offspring sex (if $P>0.05$ ). Marginal and conditional $R^{2}$ for final models are shown. Significant terms are in bold

\begin{tabular}{|c|c|c|c|c|c|c|}
\hline Sources of variation & Estimate (SE or CIs) & $d f$ & $F$ or $\chi^{2}$ & $P$ & $R_{\mathrm{m}}^{2} / R_{\mathrm{c}}^{2}$ & Var. prop \\
\hline Log-transformed body mass on day $2(N=208)$ & & & & & $0.01 / 0.14$ & \\
\hline Intercept & $0.971(0.048)$ & & & & & \\
\hline Maternal immunisation & $0.012(0.059)$ & $1,24.0$ & 0.04 & 0.85 & & \\
\hline Offspring sex & $0.075(0.042)$ & $1,198.0$ & 3.02 & 0.08 & & \\
\hline Nest of origin & $0.113(0.048,0.168)$ & 1 & 8.33 & 0.004 & & 0.13 \\
\hline Residual & $0.291(0.263,0.323)$ & & & & & 0.86 \\
\hline Body mass on day $14(N=164)$ & & & & & $0.31 / 0.76$ & \\
\hline Intercept & $17.248(0.437)$ & & & & & \\
\hline Maternal immunisation & $-0.692(0.397)$ & $1,8.2$ & 0.22 & 0.65 & & \\
\hline Brood size manipulation & $-1.926(0.555)$ & $1,18.0$ & 6.90 & 0.017 & & \\
\hline Offspring sex & $0.458(0.191)$ & $1,139.4$ & 22.82 & $<0.001$ & & \\
\hline Body mass on day $2_{\text {Within-nest effect }}$ & $0.487(0.082)$ & $1,138.6$ & 34.04 & $<0.001$ & & \\
\hline Maternal immunisation $\times$ brood size manipulation & $1.047(0.384)$ & $1,137.4$ & 6.98 & 0.009 & & \\
\hline Maternal immunisation $\times$ offspring sex & $0.592(0.304)$ & $1,136.4$ & 3.75 & 0.055 & & \\
\hline Nest of origin & $0.363(0.000,0.800)$ & 1 & 1.38 & 0.24 & & 0.04 \\
\hline Nest of rearing & $1.147(0.756,1.577)$ & 1 & 29.06 & $<0.001$ & & 0.40 \\
\hline Residual & $0.884(0.778,0.991)$ & & & & & 0.24 \\
\hline Tarsus length $(N=164)$ & & & & & $0.17 / 0.37$ & \\
\hline Intercept & $19.976(0.200)$ & & & & & \\
\hline Maternal immunisation & $-0.783(0.228)$ & 1. 11.4 & 5.13 & 0.044 & & \\
\hline Brood size manipulation & $-0.489(0.231)$ & $1,15.7$ & 0.28 & 0.61 & & \\
\hline Offspring sex & $0.415(0.122)$ & $1,148.7$ & 11.25 & 0.001 & & \\
\hline Body mass on day $2_{\text {Within-nest effect }}$ & $0.140(0.066)$ & $1,141.2$ & 4.33 & 0.039 & & \\
\hline Maternal immunisation $\times$ brood size manipulation & $0.763(0.285)$ & $1,127.7$ & 6.67 & 0.011 & & \\
\hline Nest of origin & $0.218(0.000,0.421)$ & 1 & 1.15 & 0.28 & & 0.06 \\
\hline Nest of rearing & $0.339(0.092,0.527)$ & 1 & 5.35 & 0.021 & & 0.14 \\
\hline Residual & $0.725(0.641,0.819)$ & & & & & 0.63 \\
\hline Wing length $(N=164)$ & & & & & $0.56 / 0.81$ & \\
\hline Intercept & $47.499(0.693)$ & & & & & \\
\hline Maternal immunisation & $-0.473(0.642)$ & $1,11.0$ & 0.51 & 0.49 & & \\
\hline Brood size manipulation & $-1.980(0.786)$ & $1,14.0$ & 6.05 & 0.028 & & \\
\hline Offspring sex & $0.616(0.288)$ & $1,139.7$ & 4.52 & 0.035 & & \\
\hline Body mass on day $2_{\text {Within-nest effect }}$ & $2.967(0.156)$ & $1,140.7$ & 355.02 & $<0.001$ & & \\
\hline Nest of origin & $1.208(0.574,1.934)$ & 1 & 13.31 & $<0.001$ & & 0.10 \\
\hline Nest of rearing & $1.519(0.788,2.277)$ & 1 & 18.13 & $<0.001$ & & 0.16 \\
\hline Residual & $1.670(1.479,1.876)$ & & & & & 0.19 \\
\hline
\end{tabular}

explained was the same for the nest of origin (17\%) and the nest of rearing (17\%, Table 2). Similar to the breathing rate, the aggression of offspring from enlarged broods was lower than that of offspring from unmanipulated broods (Table 2, Fig. 4). Furthermore, offspring aggression was positively correlated with body mass on day 2 (both between- and within-nest effects, Table 2). The rearing nest explained a non-significant $6 \%$ of the variance in offspring aggression (Table 2). Female offspring were more docile than male offspring $(-0.33 \pm 0.06$ vs. $-0.46 \pm 0.05,-1 \times$ no. struggles per 


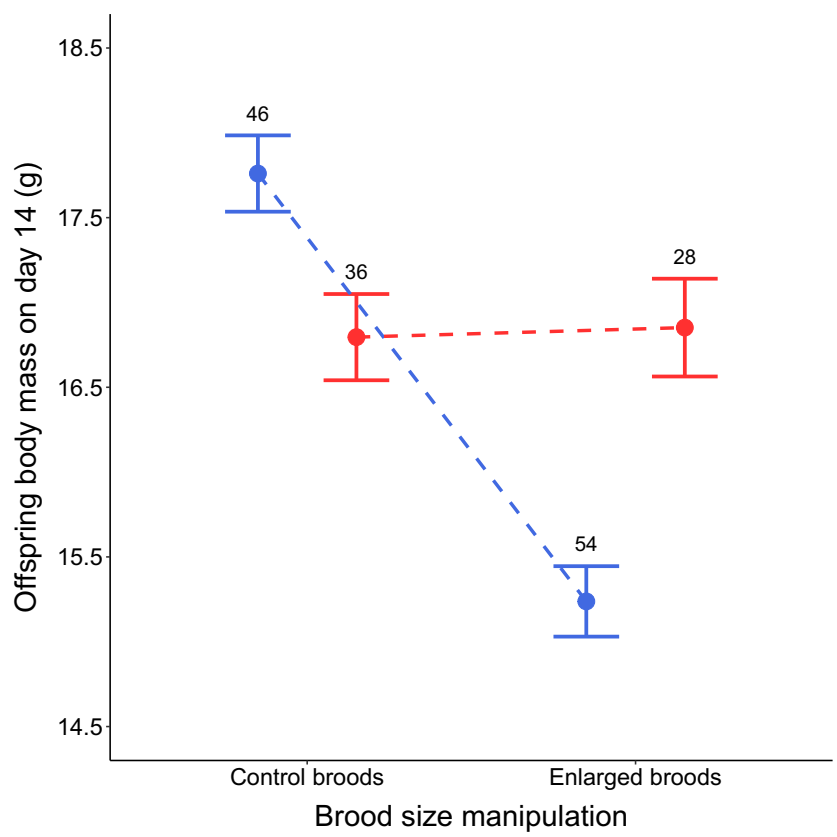

Fig. 1 Offspring body mass on day 14 in relation to maternal immunisation (control females [blue] vs. immunised females [red]) and brood size manipulation. Raw means with SEs are shown. Sample sizes are noted above bars

sec., respectively; Table 2). The degree of docility expression in 14-day-old nestlings was negatively correlated with body mass on day 2 and positively correlated with body mass on day 14 (both as within-nest effects, Table 2). Only the nest of origin explained $11 \%$ of the offspring docility variance, but this term was not significant (Table 2). We found a negative correlation between offspring aggression and docility $(r=-$ $0.48, P<0.001, N=178$ ), a weak positive correlation between aggression and breathing rate $(r=0.15, P=0.04, N=178)$, and a non-significant correlation between breathing rate and docility $(r=-0.06, P=0.43, N=178)$.

\section{Discussion}

To our knowledge, this is the first study to examine the mutual influence of immune-mediated maternal effects and posthatching rearing conditions on the development of offspring behavioural traits in birds. Contrary to our predictions, we did not detect an interaction between maternal immunisation and brood size manipulation, which could explain the variation in the examined behavioural traits of the fledged offspring. A lack of such interaction was primarily caused by the fact that we found no effect of maternal immune challenge on offspring behaviour. This result was surprising because we detected that maternal treatment and brood size manipulation interacted to affect body mass and size of 14-day-old offspring.

We found that brood enlargement negatively affected body mass and tarsus length of offspring that hatched from the eggs

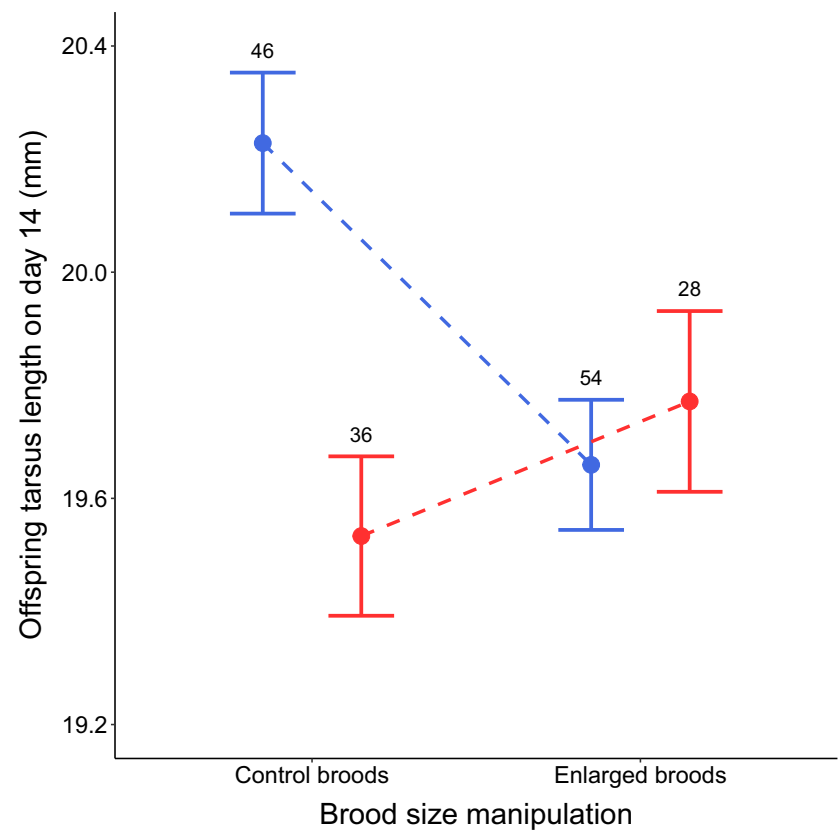

Fig. 2 Offspring tarsus length on day 14 in relation to maternal immunisation (control females [blue] vs. immunised females [red]) and brood size manipulation. Raw means with SEs are shown. Sample sizes are noted above bars

of control females. This indicated that our brood size manipulation was an efficient procedure for varying nutritional and competitive conditions among nests. These findings were also concordant with the results of previous studies indicating that harsh rearing conditions resulting from increased brood size suppressed offspring growth and led to a lower body mass and shorter tarsus length at fledging (e.g. Hõrak et al. 1999; Dubiec et al. 2006; Martyka et al. 2018). However, in the case of offspring of immunised females, brood size manipulation did not affect either body mass or tarsus length. Moreover, we revealed that offspring originating from immunised mothers tended to be heavier than offspring of control mothers when reared under harsh conditions, but not when they were reared in a favourable nest environment. However, offspring of immunised mothers had shorter tarsi compared to offspring of control mothers when reared in control broods, but this effect did not occur in enlarged broods. These findings suggested that offspring of immunised females benefited from maternal treatment only when they were reared under harsh conditions. The costs of the immune response to LPS exposure during offspring development, mainly caused by suppressing body mass gain, might be higher in poor than in favourable nutritional conditions. Support for this idea comes from studies examining the trade-off between growth and immune function in avian offspring (Hõrak et al. 1999; Brommer 2004). Maternally derived immune agents (i.e. antibodies) to offspring via eggs can help them cope with infection and thus reduce the adverse effects of developmental immunisation on growth (Grindstaff 2008). However, this may be especially 
Table 2 Results of linear mixed models examining docility, logtransformed breathing rate, and square root-transformed aggression of offspring on day 14 after hatching. Full models included maternal immunisation (control vs. immunised females), brood size manipulation (control vs. enlarged broods), offspring sex (females vs. males), and a measure of order (11 levels) as categorical fixed factors. Moreover, standardised between- and within-nest effects of nestling body mass on days 2 and 14 were entered into models as covariates. Nest of origin and rearing nest were introduced in all models as random factors (estimates of each variance component are shown accompanied with CIs and the proportion of variance explained). Presented are reduced (final) models after removing non-significant covariates and two-way interactions among maternal immunisation, brood size manipulation, and offspring sex (if $P$ $>0.05$ ). Marginal and conditional $R^{2}$ for final models are shown. Significant terms are in bold. *Omitted values because of the 11 levels of the factor

\begin{tabular}{|c|c|c|c|c|c|c|}
\hline Sources of variation & Estimate (SE or CIs) & $d f$ & $F$ or $\chi^{2}$ & $P$ & $R_{\mathrm{m}}^{2} / R_{\mathrm{c}}^{2}$ & Var. prop \\
\hline Docility $(N=164)$ & & & & & $0.26 / 0.37$ & \\
\hline Intercept & $-0.246(0.112)$ & & & & & \\
\hline Maternal immunisation & $-0.127(0.090)$ & $1,19.7$ & 1.88 & 0.19 & & \\
\hline Brood size manipulation & $0.057(0.071)$ & $1,10.1$ & 0.54 & 0.48 & & \\
\hline Offspring sex & $-0.184(0.067)$ & $1,133.2$ & 7.12 & 0.009 & & \\
\hline Measure order & $*$ & $10,131.6$ & 1.45 & 0.17 & & \\
\hline Body mass on day $2_{\text {Within-nest effect }}$ & $-0.264(0.040)$ & $1,138.6$ & 41.95 & $<0.001$ & & \\
\hline Body mass on day $14_{\text {Within-nest effect }}$ & $0.131(0.044)$ & $1,136.7$ & 8.77 & 0.004 & & \\
\hline Nest of origin & $0.154(0.000,0.233)$ & 1 & 3.60 & 0.058 & & 0.11 \\
\hline Nest of rearing & $0.000(0.000,0.167)$ & & & & & 0.00 \\
\hline Residual & $0.368(0.313,0.396)$ & & & & & 0.63 \\
\hline Log-transformed breathing rate $(N=175)$ & & & & & $0.19 / 0.53$ & \\
\hline Intercept & $0.530(0.055)$ & & & & & \\
\hline Maternal immunisation & $-0.006(0.046)$ & 1.13 .1 & 0.01 & 0.91 & & \\
\hline Brood size manipulation & $-0.116(0.049)$ & $1,12.8$ & 5.23 & 0.040 & & \\
\hline Offspring sex & $-0.051(0.025)$ & $1,141.3$ & 3.92 & 0.050 & & \\
\hline Measure order & $*$ & $10,138.7$ & 1.47 & 0.16 & & \\
\hline Body mass on day $14_{\text {Within-nest effect }}$ & $0.064(0.013)$ & $1,141.5$ & 22.37 & $<0.001$ & & \\
\hline Nest of origin & $0.084(0.036,0.138)$ & 1 & 8.93 & 0.003 & & 0.17 \\
\hline Nest of rearing & $0.085(0.027,0.136)$ & 1 & 5.78 & 0.016 & & 0.17 \\
\hline Residual & $0.141(0.121,0.152)$ & & & & & 0.47 \\
\hline Square root-transformed aggression $(N=164)$ & & & & & $0.38 / 0.44$ & \\
\hline Intercept & $1.347(0.070)$ & & & & & \\
\hline Maternal immunisation & $0.055(0.054)$ & $1,18.5$ & 0.10 & 0.34 & & \\
\hline Brood size manipulation & $-0.152(0.047)$ & $1,11.8$ & 9.12 & 0.011 & & \\
\hline Offspring sex & $0.063(0.041)$ & $1,139.3$ & 2.25 & 0.14 & & \\
\hline Measure order & $*$ & $10,133.6$ & 1.52 & 0.14 & & \\
\hline Body mass on day $2_{\text {Between-nest effect }}$ & $0.112(0.029)$ & $1,19.9$ & 13.78 & 0.001 & & \\
\hline Body mass on day $2_{\text {Within-nest effect }}$ & $0.135(0.022)$ & $1,138.2$ & 34.64 & $<0.001$ & & \\
\hline Nest of origin & $0.076(0.000,0.121)$ & 1 & 1.60 & 0.21 & & 0.06 \\
\hline Nest of rearing & $0.000(0.000,0.095)$ & & & & & 0.00 \\
\hline Residual & $0.241(0.206,0.261)$ & & & & & 0.56 \\
\hline
\end{tabular}

advantageous when offspring are reared under harsh conditions, but not if such conditions are 'optimal' (Martyka et al. 2018). The differences in patterns of body mass and tarsus length in relation to maternal immunisation and brood size manipulation may indicate different strategies of resource allocation. Body mass gain and skeletal growth of offspring, at least until fledging, may be prioritised differentially, depending on maternal and offspring exposure to an antigen and brood size during nestling development (Grindstaff et al.
2012; Martyka et al. 2018). Overall, these results confirmed earlier findings that the potential influence of immunemediated maternal effects on offspring phenotype might be modulated by various post-hatching rearing conditions (Ismail et al. 2015; Martyka et al. 2018). The observed effects of maternal immunisation on offspring body mass and size seem to come primarily from differences in egg composition; mostly antibodies but also hormones might be involved (Bowers et al. 2015). We found no effects of maternal immune 


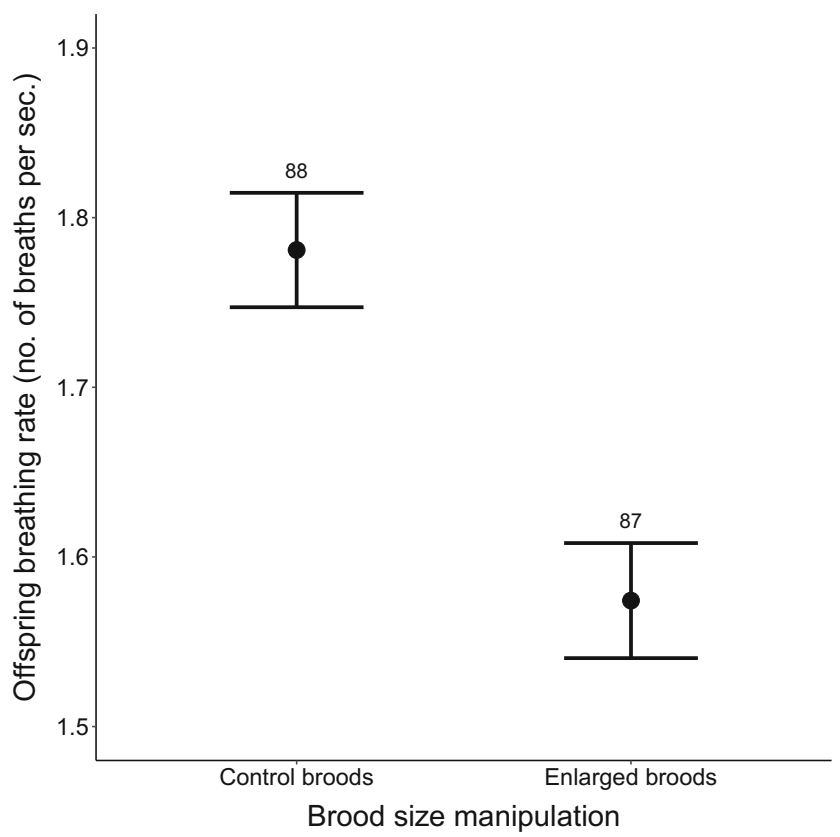

Fig. 3 Offspring breathing rate in relation to brood size manipulation. Raw means with SEs are shown. Sample sizes are noted above bars

challenge on primary reproductive effort. Additionally, we partially cross-fostered nestlings among the broods of immunised and control females. Thus, it is unlikely that our results were biased because of potential carry-over effects resulting from maternal immunisation.

As previously shown, immune-mediated maternal effects and post-natal rearing conditions can play a mutual role in determining offspring body mass and size (present study; Ismail et al. 2015; Martyka et al. 2018). Therefore, the question is why we failed to detect the effects of maternal treatment on offspring behaviour. First, this result may suggest that nestling exposure to LPS did not affect offspring neurodevelopment to such an extent that allowed the detection of potential changes in behavioural traits as a result of maternal immune challenge. However, our research was limited to recognising the short-term effects of treatment on offspring behaviour. Therefore, we cannot exclude the possibility that potential effects of maternal immunisation, which were expected to modify the impact of offspring exposure to LPS on behaviour, could appear later in life. Adult birds can more actively respond to environmental and social stressors than nestlings, indicating that the expression of behavioural traits may vary between successive stages of life. Indeed, different behavioural responses to the environment can have distinct adaptive values at different periods in life or across environmental contexts (Naguib et al. 2011; Groothuis and Taborsky 2015). Therefore, even though environmental circumstances can have a significant influence on behavioural development, such factors still may not lead to immediate consequences for offspring behaviour at the fledgling stage; instead, they may be delayed in time and ultimately revealed in adulthood. Second, maternal treatment would not affect offspring behaviour if nestling

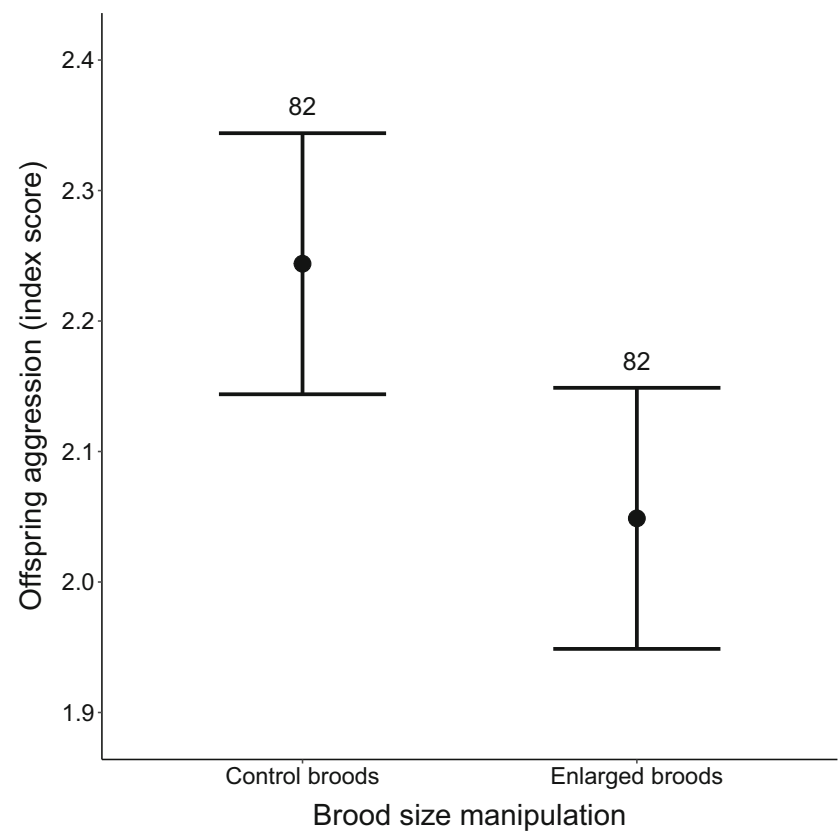

Fig. 4 Offspring aggression in relation to brood size manipulation. Raw means with SEs are shown. Sample sizes are noted above bars

exposure to LPS does not provide a reliable cue for adult life conditions. It has been hypothesised that early developmental conditions may have long-lasting consequences for organisms only when they reliably predict future adult environments (Bateson et al. 2014). Third, even though we found that maternal immunisation and brood size manipulation interactively affected offspring growth, we cannot exclude that such interactive effects might not be passed on offspring behaviour in a straight way. For example, Grindstaff et al. (2012) found that maternal and developmental immunisation interactively affected body mass gain in offspring, but the observed pattern of body mass changes did not reflect alterations observed in behaviour after the treatments. This suggests that the development of morphological and behavioural traits are followed, to some extent, by different mechanisms, and there is no simple connection between morphological developmental trajectories and neurodevelopment. Furthermore, we examined only three easily tested behavioural traits without looking at other traits potentially sensitive to immune activation. To date, offspring exposed to LPS during early life has been shown to have a lower potential for learning and memorisation, and this was found both in birds (Grindstaff et al. 2012) and mammals (Bilbo and Schwarz 2009). Very few studies have examined the effects of maternal or neonatal immune challenges on the development and expression of behavioural traits in avian offspring, and those studies that exist do not show a common pattern. For example, in the same study on zebra finches, the authors showed that nestling exposure to LPS caused males to be more neophobic than females if their mothers were previously immunised with antigens other than LPS, but there were no such sex differences in neophobia in offspring if mothers were immunised with LPS or came from the control group 
(Grindstaff et al. 2012). Additionally, mechanisms underlying the process linked to immune activation and its effects on neurodevelopment and, consequently, behavioural expression are poorly understood (Grindstaff 2016). These facts make it difficult to interpret our results and lead to highly speculative explanations.

Interestingly, our study demonstrated that manipulating posthatching rearing conditions independently affected offspring behaviour. We found that two of the three traits examined in offspring, i.e. breathing rate and aggression, were shaped by brood size manipulation. In contrast, docility was not affected by this treatment. The observed effects of brood enlargement on the breathing rate and offspring aggression were the opposite of previous research results. Earlier studies have shown that adverse rearing conditions during nestling development (i.e. poor nutrition) increase rather than decrease the level of physiological stress responses (Kitaysky et al. 2001), handling stress (van Oers et al. 2015; but see Naguib et al. 2011), and aggression in the offspring (Carere et al. 2005). In contrast, we found that offspring reared under harsh rearing conditions exhibited lower breathing rates and aggression than offspring reared in unmanipulated broods. These are puzzling results because it is expected that offspring reared in a competitive environment should be more aggressive, allowing them to take more resources during development and adapt to similar environmental conditions in the future (Carere et al. 2005; Naguib et al. 2011). However, increased aggression, especially under highly competitive rearing conditions, may not create benefits for individuals when the among-individual competition is lower (Duckworth 2006). Therefore, in particular environmental or social circumstances, individuals may be expected to alter their aggression level such that the potential costs do not exceed the benefits of that behaviour. Similarly, breathing rate should also be higher when rearing conditions are harsh, as increased competition for food may generate substantial stress among siblings, partially as a result of enhanced aggression. Breathing rate as a measure of offspring handling stress is a positive predictor of exploratory behaviour in adult life (Fucikova et al. 2009). Thus, if nestlings encounter poor nutritional conditions during development and there is a match with subsequent environmental conditions occurring in their adult life, then the higher level of stress experienced during ontogeny would be adaptive and provide long-lasting fitness benefits (Bateson et al. 2014; Groothuis and Taborsky 2015). However, this was not the case, and we found a reverse pattern in breathing rate in relation to brood size enlargement in our study. Notably, the lower breathing rate and aggression among offspring from broods containing more nestlings may result from the feeding behaviour of parents raising enlarged broods. For instance, they could change the quality or quantity of food items delivered to offspring, which might affect the brain development of offspring and ultimately their stress response and/or aggression. García-Navas and Sanz (2010) found that blue tit Cyanistes caeruleus parents raising nestlings in enlarged broods provisioned them with a significantly larger number of caterpillars and a smaller number of spiders than parents in control or reduced broods. Among arthropods, spiders are the primary source of taurine (Ramsay and Houston 2003), that is, sulfonated amino acid, which plays a role in neuronal development and influences behavioural trait expression (Franconi et al. 2004). Indeed, Arnold et al. (2007) experimentally manipulated the taurine level provided to offspring during development and showed that nestlings supplemented with a higher amount of taurine exhibited lower neophobia and greater risk-taking behaviour. In turn, van Oers et al. (2015) documented that the biomass of caterpillars, but not spiders, affected the level of handling stress in great tit offspring, indicating that nestlings provisioned by a larger number of caterpillars were less stressed. Thus, we speculate that differences in diet resulting from the response of parents to brood size may be significant to the establishment of some behavioural traits. However, Naguib et al. (2011) demonstrated that great tit offspring reared in smaller broods exhibited higher stress levels than those reared in normal-sized broods. The authors suggested that this result may be caused by different parental feeding behaviour patterns, as we suggest above, higher thermoregulatory costs, or more space for sibling competition regarding parental care. We have a similar situation in which unmanipulated broods are numerically smaller than enlarged broods; thus, some of those proposed explanations could also be applied to our study results.

In conclusion, our experimental study did not detect the effects of maternal immunisation on offspring behaviour; thus, we were also unable to find any interactive effects of maternal immune challenge and brood size manipulation. We found that two of the studied behavioural traits were affected by brood enlargement. Because of the limited number of studies testing the mutual influence of immune-mediated maternal effects and the post-hatching rearing environment on behavioural trait expression under natural conditions in birds, our study contributes to better understanding of the processes that underlie the shaping of an individual's behaviour.

Supplementary Information The online version contains supplementary material available at https://doi.org/10.1007/s00265-021-02995-5.

Acknowledgements We thank anonymous referees for critical comments to an earlier version of this manuscript. We also thank Agata Rozik for assistance with fieldwork.

Author contribution RM conceived and planned the study, conducted the experiment, collected data in the field, performed statistical analyses, interpreted results, and wrote the first draft of the manuscript; EBŚ performed molecular sex determination of the offspring; EBS and PT contributed to the interpretation of the results and writing of the manuscript. All authors read and approved the final manuscript.

Funding The National Science Centre (Poland) financed this study via a post-doctoral fellowship (grant number: DEC-2012/04/S/NZ8/00213). The Institute of Nature Conservation of the Polish Academy of 
Sciences partially supported this research via statutory funds (DS III.1/ 2020).

Data availability The dataset generated and analysed during the study is available as supplementary material.

Code availability Not applicable.

\section{Declarations}

Ethics approval We followed all applicable international, national, and institutional guidelines for the use of animals. The Poznan Local Ethics Committee for Animal Experimentation approved the experimental procedures conducted in our study (permit number: 72/2012). The Regional (Kraków) and General Directorate for Environmental Protection in Poland permitted to conduct this study in wild bird population (permit numbers: OP-I.6401.394.2012.MMr and DOP-OZ.6401.03.18.2013.dł).

\section{Consent to participate Not applicable.}

Consent for publication Not applicable.

Conflict of interest The authors declare no competing interest.

Open Access This article is licensed under a Creative Commons Attribution 4.0 International License, which permits use, sharing, adaptation, distribution and reproduction in any medium or format, as long as you give appropriate credit to the original author(s) and the source, provide a link to the Creative Commons licence, and indicate if changes were made. The images or other third party material in this article are included in the article's Creative Commons licence, unless indicated otherwise in a credit line to the material. If material is not included in the article's Creative Commons licence and your intended use is not permitted by statutory regulation or exceeds the permitted use, you will need to obtain permission directly from the copyright holder. To view a copy of this licence, visit http://creativecommons.org/licenses/by/4.0/.

\section{References}

Arct A, Drobniak SM, Podmokła E, Gustafson L, Cichoń M (2013) Benefits of extra-pair mating may depend on environmental conditions - an experimental study in the blue tit (Cyanistes caeruleus). Behav Ecol Sociobiol 67:1809-1815

Arnold KE, Ramsay SL, Donaldson C, Adam A (2007) Parental prey selection affects risk-taking behaviour and spatial learning in avian offspring. Proc R Soc Lond B 274:2563-2569

Bartoń K (2020) MuMIn: Multi-Model Inference. R package version 1(43):17 https://CRAN.R-project.org/package=MuMIn

Bates D, Maechler M, Bolker B, Walker S (2015) Fitting linear mixedeffects models using lme4. J Stat Softw 67:1-48

Bateson P, Gluckman P, Hanson M (2014) The biology of developmental plasticity and the predictive adaptive response hypothesis. J Physiol 592:2357-2368

Bilbo SD, Schwarz JM (2009) Early-life programming of later-life brain and behaviour: a critical role for the immune system. Front Behav Neurosci 3:14

Bischoff LL, Tschirren B, Richner H (2009) Long-term effects of early parasite exposure on song duration and singing strategy in great tits. Behav Ecol 20:265-270
Boulanger-Bertolus J, Pancaro C, Mashour GA (2018) Increasing role of maternal immune activation in neurodevelopmental disorders. Front Behav Neurosci 12:230

Bowers EK, Bowden RM, Sakaluk SK, Thompson CF (2015) Immune activation generates corticosterone-mediated terminal reproductive investment in a wild bird. Am Nat 185:769-783

Brommer JE (2004) Immunocompetence and its costs during development: an experimental study in blue tit nestlings. Proc R Soc Lond B 271:S110-S113

Brommer JE, Kluen E (2012) Exploring the genetics of nestling personality traits in a wild passerine bird: testing the phenotypic gambit. Ecol Evol 2:3032-3044

Butler MW, Toomey MB, McGraw KJ, Rowe M (2012) Ontogenetic immune challenges shape adult personality in mallard ducks. Proc R Soc Lond B 279:326-333

Careau V, Buttemer WA, Buchanan KL (2014) Early-developmental stress, repeatability, and canalization in a suite of physiological and behavioural traits in female zebra finches. Integr Comp Biol 54:539-554

Carere C, Maestripieri D (2013) Animal personalities: behaviour, physiology and evolution. University of Chicago Press, Chicago

Carere C, van Oers K (2004) Shy and bold great tits (Parus major): body temperature and breath rate in response to handling stress. Physiol Behav 82:905-912

Carere C, Drent PJ, Koolhaas JM, Groothuis TGG (2005) Epigenetic effects on personality traits: early food provisioning and sibling competition. Behaviour 142:1329-1355

David M, Auclair Y, Cézilly F (2011) Personality predicts social dominance in female zebra finches, Taeniopygia guttata, in a feeding context. Anim Behav 81:219-224

Drent PJ, van Oers K, van Noordwijk AJ (2003) Realized heritability of personalities in the great tit (Parus major). Proc R Soc Lond B 270: $45-51$

Dubiec A, Cichoń M, Deptuch K (2006) Sex-specific development of cell-mediated immunity under experimentally altered rearing conditions in blue tit nestlings. Proc R Soc Lond B 273:1759-1764

Duckworth RA (2006) Behavioral correlations across breeding context provide a mechanism for a cost of aggression. Behav Ecol 17:10111019

Fischer S, Bessert-Nettelbeck M, Kotrschal A, Taborsky B (2015) Rearing group size determines social competence and brain structure in a cooperatively breeding cichlid. Am Nat 186:123-140

Franconi F, Diana G, Fortuna A, Galietta G, Trombetta G, Valentini G, Seghieri G, Loizzo A (2004) Taurine administration during lactation modifies hippocampal CA1 neurotransmission and behavioural programming in adult male mice. Brain Res Bull 63:491-497

Fucikova E, Drent PJ, Smits N, van Oers K (2009) Handling stress as a measurement of personality in great tit nestlings (Parus major). Ethology 115:366-374

García-Navas V, Sanz JJ (2010) Flexibility in the foraging behaviour of blue tits in response to short-term manipulation of brood size. Ethology 116:744-754

Gosler A (1993) The great tit. Hamlyn, London

Griffiths R, Double MC, Orr K, Dawson RJG (1998) A DNA test to sex most birds. Mol Ecol 7:1071-1075

Grindstaff JL (2008) Maternal antibodies reduce costs of an immune response during development. J Exp Biol 211:654-660

Grindstaff JL (2016) Developmental immune activation programs adult behaviour: insight from research on birds. Curr Opin Behav Sci 7: $21-27$

Grindstaff JL, Hasselquist D, Nilsson JA, Sandell M, Smith HG, Stjernman M (2006) Transgenerational priming of immunity: maternal exposure to a bacterial antigen enhances offspring humoral immunity. Proc R Soc Lond B 273:2551-2557 
Grindstaff JL, Hunsaker VR, Cox SN (2012) Maternal and developmental challenges alter behaviour and learning ability of offspring. Horm Behav 62:337-344

Groothuis TGG, Taborsky B (2015) Introducing biological realism into the study of developmental plasticity in behaviour. Front Zool 12:S6

Halekoh U, Hojsgaard S (2014) A Kenward-Roger approximation and parametric bootstrap methods for tests in linear mixed models - the R package pbkrtest. J Stat Softw 59:1-30

Hart BL (1988) Biological basis of the behavior of sick animals. Neurosci Biobehav Rev 12:123-137

Hõrak P, Tegelmann L, Ots I, Møller AP (1999) Immune function and survival of great tit nestlings in relation to growth conditions. Oecologia 121:316-322

Ismail A, Jacquin L, Haussy C, Perret S, Gasparini J (2015) Food availability modulates the effects of maternal antibodies on growth and immunity in young feral pigeons. J Avian Biol 46:489-494

Karrow NA (2006) Activation of the hypothalamic-pituitary-adrenal axis and autonomic nervous system during inflammation and altered programming of the neuroendocrine-immune axis during fetal and neonatal development: lessons learned from the model inflammagen, lipopolysaccharide. Brain Behav Immun 20:144-158

Kasumovic MM (2013) The multidimensional consequences of the juvenile environment: towards an integrative view of the adult phenotype. Anim Behav 85:1049-1059

Kitaysky AS, Kitaiskaia AS, Wingfield JC, Piatt JF (2001) Dietary restriction causes chronic elevation of corticosterone and enhances stress response in red-legged kittiwake chicks. J Comp Physiol B 171:701-709

Klasing KC, Leshchinsky TV (1999) Functions, costs, and benefits of the immune system during development and growth. In: Adams NJ, Slotow RH (eds) 22nd International ornithological congress. BirdLife, South Africa, pp 2817-2835

Krause ET, Naguib M (2011) Compensatory growth affects exploratory behaviour in zebra finches, Taeniopygia guttata. Anim Behav 81: $1295-1300$

Kuznetsova A, Brockhoff PB, Christensen RHB (2017) lmerTest package: tests in linear mixed effects models. J Stat Softw 82:1-26

Laine VN, Gossmann TI, Schachtschneider KM et al (2016) Evolutionary signals of selection on cognition from the great tit genome and methylome. Nat Commun 7:10474

Langenhof MR, Komdeur J (2018) Why and how the early-life environment affects development of coping behaviours. Behav Ecol Sociobiol 72:34

Lenth R (2020) emmeans: estimated marginal means, aka least-squares means. R package version 1.3.4, https://CRAN.R-project.org/ package $=$ emmeans

Lindström J (1999) Early development and fitness in birds and mammals. Trends Ecol Evol 14:343-348
Lüdecke D, Waggoner P, Makowski D (2019) insight: a unified interface to access information from model objects in R. J Open Source Softw $4: 1412$

Martyka R, Śliwińska EB, Martyka M, Cichoń M, Tryjanowski P (2018) The effect of pre-laying maternal immunization on offspring growth and immunity differs across experimentally altered postnatal rearing conditions in a wild songbird. Front Zool 15:25

Merrill L, Grindstaff JL (2014) Maternal antibody transfer can lead to suppression of humoral immunity in developing zebra finches (Taeniopygia guttata). Physiol Biochem Zool 87:740-751

Møller AP, Simon S, Christiansen SS, Timothy A, Mousseau TA (2011) Sexual signals, risk of predation and escape behaviour. Behav Ecol 22:800-807

Mousseau AT, Fox CW (1998) Maternal effects as adaptations. Oxford University Press, New York

Naguib M, Flörcke C, van Oers K (2011) Effects of social conditions during early development on stress response and personality traits in great tits (Parus major). Dev Psychobiol 53:592-600

Noguera JC, Metcalfe NB, Surai PF, Monaghan P (2015) Are you what you eat? Micronutritional deficiencies during development influence adult personality-related traits. Anim Behav 101:129-140

R Core Team (2020) R: a language and environment for statistical computing. R Foundation for Statistical Computing, Vienna, https:// www.R-project.org/

Ramsay SL, Houston DC (2003) Amino acid composition of some woodland arthropods and its implications for breeding tits and other passerines. Ibis 145:227-232

Riebel K, Spierings MJ, Holveck MJ, Verhulst S (2012) Phenotypic plasticity of avian social-learning strategies. Anim Behav 84: $1533-1539$

Stamps J, Groothuis TGG (2010) The development of animal personality: relevance, concepts and perspectives. Biol Rev 85:301-325

Sunwoo HH, Nakano T, Dixon WT, Sim JS (1996) Immune responses in chickens against lipopolysaccharide of Escherichia coli and Salmonella typhimurium. Poult Sci 75:342-345

Trillmich F, Müller T, Müller C (2018) Understanding the evolution of personality requires the study of mechanisms behind the development and life history of personality traits. Biol Lett 14:20170740

van de Pol M, Wright J (2009) A simple method for distinguishing within- versus between-subject effects using mixed models. Anim Behav 77:753-758

van Oers K, Kohn GM, Hinde CA, Naguib M (2015) Parental food provisioning is related to nestling stress response in wild great tit nestlings: implications for the development of personality. Front Zool 12:S10

Publisher's note Springer Nature remains neutral with regard to jurisdictional claims in published maps and institutional affiliations. 